of Life Technologies in Carlsbad, California. Within a day, Wells can detect duplications or deletions of entire chromosomes - the abnormalities seen most often early in development. Several women are now pregnant with embryos selected using this technique, and a larger clinical trial is planned for later this year. Wells says that the low cost - as much as 50\% less than current methods - should open up testing to more women.

Other researchers are refining existing technologies to increase the average length of the DNA strand that can be 'read' from the sequencer. The devices that dominate the field sequence short fragments of DNA, no more than a few hundred base pairs, and overlook some portions of the genome. Longer reads are needed, for example, to distinguish between closely related microorganisms - an important requirement for researchers studying whether overabundance of some microbial species in the gut or vagina make patients more susceptible to problems ranging from diarrhoea to premature birth.

Late last year, Illumina bought Moleculo, a company based in San Francisco, California, whose technology extends reads by changing the way that DNA is prepared for sequencing and by applying new analytical software to the readout. These tricks can coax reads thousands

\section{REASONABLY PRICED GENOMES}

Although no reports of big innovations in DNA sequencing are expected at a major conference this week the current cost and capabilities of the technology now make medical applications worthwhile.

\begin{tabular}{l|l|l|l|} 
Name & Machine cost & Read length (bases) & Cost per megabase \\
Illumina MiSeq & US $\$ 125,000$ & 500 & $14-70$ cents \\
Illumina HiSeq & US $\$ 690,000$ & 300 & $4-5$ cents \\
PacBio RS & US $\$ 695,000$ & 4,575 & $\$ 2-17$ \\
Ion Torrent PGM & US $\$ 49,000$ & 400 & 60 cents- $\$ 5$ \\
Ion Torrent Proton & US $\$ 224,000$ & 200 & $1-9$ cents
\end{tabular}

of bases long out of Illumina machines - an order of magnitude greater than previously possible. The machines produced by Pacific Biosciences in Menlo Park, California, also achieve read lengths that routinely reach into the thousands of bases, thanks to an engineered polymerase. Customers say that the company seems to have overcome early problems with reliability.

In market share, Pacific Biosciences still has a long way to go to catch Illumina or Life Technologies, which this month said that it expects sales of the Ion Torrent sequencing system to boost revenue by up to $5 \%$ in 2013 . Corporate takeovers are a topic of fevered speculation in the sequencing world. Rumours that Life Technologies' sequencing business may be acquired sent its shares soaring to their highest price ever. And Illumina recently fended off a hostile takeover bid by Swiss company Roche in Basel, which formally dropped its offer a month ago. But the fundamental landscape has not changed much.

Any reshuffling of the major players will have to wait for the field's next 'tick' cycle, whether it takes the form of Life Technologies' Ion Proton machine - which is just hitting labs and which the company says will eventually deliver a $\$ 1,000$ genome in a day - or Oxford Nanopore's GridION and MinION machines, which scientists still haven't seen. In the meantime, says Shendure, "this year's meeting will be less glitz and more science, and that's great".

\title{
Dark-matter hunt gets deep
}

\section{China launches world's deepest particle-physics experiment - but it joins a crowded field.}

\section{BY EUGENIE SAMUEL REICH}

$\mathrm{M}$ ore than 1,000 metres underground, physicists have set traps of liquid xenon to catch their prey: hypothetical particles of dark matter that might very rarely interact with ordinary matter as they drift through Earth. With construction costs on the order of US $\$ 10$ million each, such experiments are a relatively cheap way to work out the composition of $85 \%$ of the matter in the Universe. But does the world really need four of them?

Ongoing experiments in Italy, the United States and Japan are now being joined by a fourth in China, called PandaX (see 'Dark and deep'). Installed in the deepest laboratory in the world, 2,500 metres under the marble mountain of JinPing in Sichuan province, PandaX will this year begin monitoring 120 kilograms of xenon. The team hopes to scale the tank up to 1 tonne by 2016 , which would mean that the experiment had developed more quickly than any other dark-matter search. "We want to demonstrate

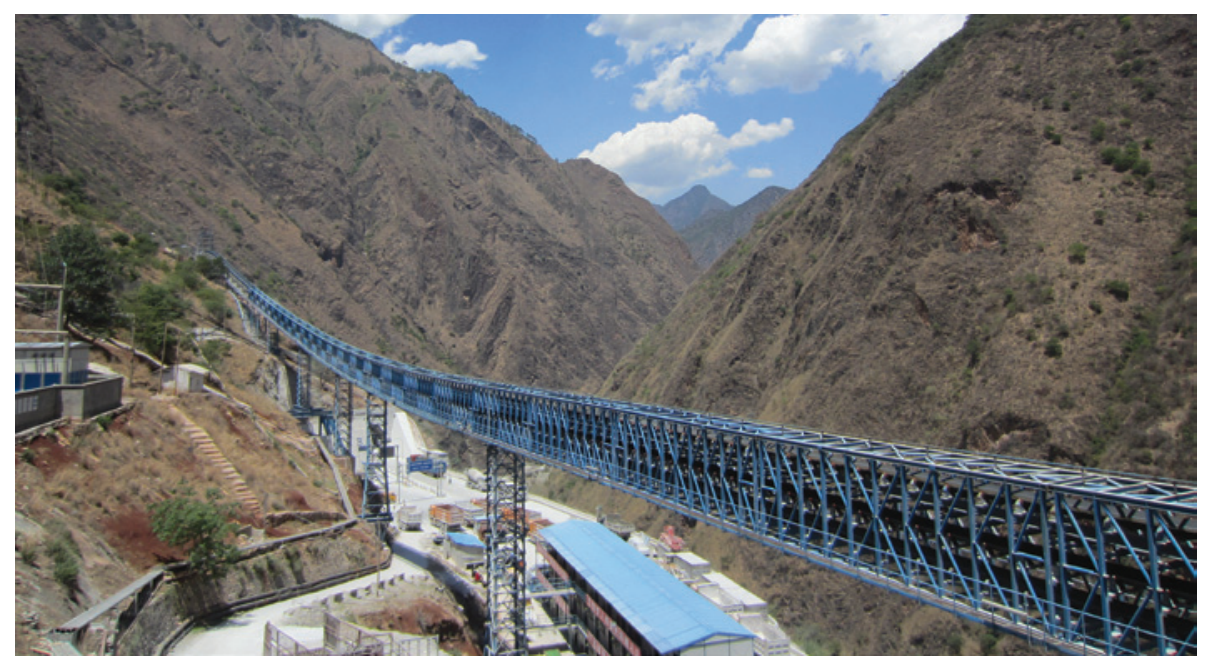

A conveyor belt removes rock from JinPing laboratory, a 2,500-metre-deep dark-matter experiment site.

that world-class research in dark matter is possible in China," says Xiangdong Ji, a physicist at Shanghai Jiao Tong University in China and a spokesman for PandaX.
Dark-matter researchers in the West are excited by the ambition of the project, but some question the duplication of effort. "Spending all our money on different direct-detection 


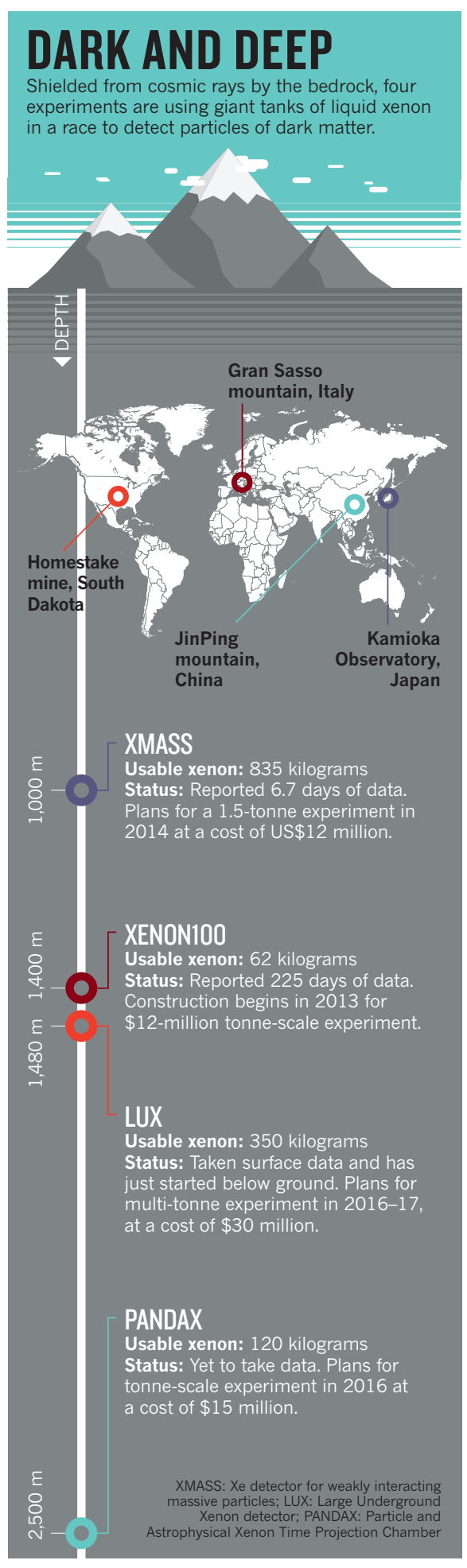

> experiments is not worth it," says Stefan Funk, an astrophysicist at the SLAC National Accelerator Laboratory in Menlo Park, California, who admits that he is biased - he looks for dark matter indirectly, by watching the heavens for a possible $\gamma$-ray signal.

The proliferation of xenon experiments reflects infighting among the dark-matter hunters, as well as national ambitions to be first to answer one of physics' most compelling questions. Observations of the rotation of galaxies and of the cosmic background radiation - the afterglow of the Big Bang — suggest that some $85 \%$ of matter in the Universe exerts gravitational pull, but does not radiate light. Leading theories suggest that such dark matter comes in the form of weakly interacting massive particles (WIMPs). If they exist, these would occasionally collide with nuclei of ordinary matter, so they could in principle be directly detected in a large tank of an atomically dense material such as xenon, which produces light when a nucleus recoils.

Such experiments are placed underground to minimize interference by cosmic rays that can mimic WIMPs. So far, they have seen nothing. The most progress has been made by XENON100, an experiment near L'Aquila, Italy, which has not detected a WIMP signal in 225 days of running time so far, ruling out the existence of heavier and more strongly interacting particles. In a few months, the XENON collaboration will start to scale up its detector to more than 1 tonne of xenon, making it 100 times more sensitive.

Enter PandaX, which was born when China's National Natural Science Foundation and Ministry of Science and Technology funded a team that included members of the XENON collaboration. They set up an experiment of their own, deep within JinPing mountain, in a small laboratory that opened in 2011 after just two years of construction. PandaX hopes to rival XENON100's current sensitivity for lighter WIMPs by the end of this year, although Ji acknowledges the challenges of working in a remote area of central China while relying on overseas suppliers for many parts. Just last week, the team was unable to cool down its experiment because of a delay in the delivery of liquid nitrogen. But because PandaX is more than a kilometre deeper than the other experiments, it needs less shielding from cosmic rays, which will make it easier to scale up.

China, together with a consortium of universities including some in the United States, is spending about $\$ 15$ million to build PandaX, but the team would like more international partners. In January, PandaX applied to the US Department of Energy (DOE), which has not set up an agreement to work with the Chinese foundation, but the US agency declined. Instead, the DOE is spending its money on another group that split off from the XENON collaboration: the Large Underground Xenon (LUX) experiment at the Homestake gold mine in South Dakota.

Homestake was once meant to be the site of a major underground laboratory. But the lab's main funder, the US National Science Foundation (NSF), dropped the idea in 2010 (see Nature http://doi.org/bcbr7z; 2010), leaving the DOE to run it alone and bear the whole cost of the constant pumping required to prevent the mine from flooding.

LUX might be the most vulnerable of all the xenon experiments, says Juan Collar, a physicist at the University of Chicago in Illinois who looks for lighter WIMPs with a number of smaller experiments. "The funding situation is really terrible," he says. The DOE has indicated that it plans to reduce the number of large dark-matter experiments it funds in October, so LUX could face the axe. US contributions to XENON100 are funded through the NSF, so it won't be subject to the winnowing process.

Rick Gaitskell, a physicist at Brown University in Providence, Rhode Island, and spokesman for LUX, says that the United States needs its own deep underground lab to maintain its dark-matter expertise. "Why would we give up that leadership position and move all that experience overseas?" he asks.

But Ji, who now has access to perhaps the best underground lab in the world, says that some consolidation is inevitable. The international community is unlikely to support more than two xenon experiments with multitonne detectors, he says, and the United States will need to choose which effort to back. In the meantime, he says, it is not a bad idea to have many groups working to improve the technology. "That will help build the ultimate darkmatter experiment."
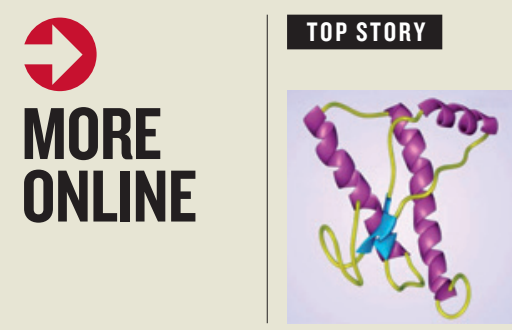

Well-folded prion proteins play 'good' role in brain development go.nature.com/ bc $47+5$

\section{MORE NEWS}

- The last of the Medici line may not have died of syphilis after all go.nature. com/gws6g3

- Tracking complex networks from a few nodes go.nature.com/092aja

- Supernova shockwaves produce cosmic rays go.nature.com/phyyf5

\section{ENVIRONMENT}

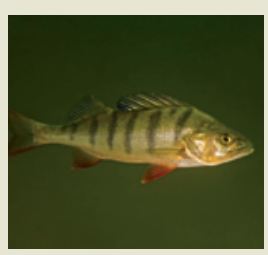

Anti-anxiety drugs increase aggression in river fish go.nature.com/ d9nloa 